EPJ Web of Conferences 41, 12015 (2013)

DOI: $10.1051 /$ epjconf/20134112015

C) Owned by the authors, published by EDP Sciences, 2013

\title{
Temporal Coherence Effects on Coherent Diffractive Imaging of a Binary Sample by a High Harmonic Source
}

\author{
A.D. Parsons ${ }^{1}$, R.T. Chapman ${ }^{2}$, B. Mills ${ }^{1}$, S. Bajt ${ }^{3}$, J.G. Frey ${ }^{2}$, W.S. Brocklesby ${ }^{1}$ \\ ${ }^{1}$ Optoelectronics Research Centre, University of Southampton, Southampton, SO17 1BJ,UK \\ ${ }^{2}$ School of Chemistry, University of Southampton, Southampton, SO17 1BJ,UK \\ ${ }^{3}$ Photon Science, DESY, Notkestrasse 85, 22607 Hamburg, Germany
}

\begin{abstract}
Coherent Diffractive Imaging (CDI) is performed with single and multiple harmonics from an ultrafast HHG source. The effect of HHG source bandwidth on the effectiveness of the reconstruction algorithms is compared. A low quality reconstruction from broadband data is achieved assuming full coherence in the algorithm.
\end{abstract}

\section{Introduction}

To investigate nano-scale structures, the Abbe diffraction limit [1] suggests that the wavelength of the probe may be decreased to improve resolution. Optically, this requires using extreme ultraviolet (EUV $(10-50 \mathrm{~nm}))$ or X-ray radiation. Many sources of such radiation exist, for example synchrotrons and X-ray free electron lasers (XFELs), however their size and cost limit accessibility and availability. To overcome this limitation two lab-based options have become mainstream: high harmonic generation (HHG), and laser plasma (LP) sources.

At EUV wavelengths, imaging optics are both expensive and technically difficult to make. Instead, it is possible to record oversampled far-field diffraction patterns from samples and retrieve the phases using iterative algorithms. A similar phase problem to that in crystallography is solved to retrieve the full E-field distribution in the object plane by a process known as coherent diffractive imaging (CDI) [2]. The coherence of the source is sampled in the signal is critical for CDI.

HHG) produces a train of spatially and temporally coherent EUV pulses $\left(1_{\text {temporal }}=90 \mathrm{um}, 1_{\text {spatial }}=\right.$ width of beam). Since the beat period between harmonics is much shorter than experimental integration times, an incoherent sum of diffraction signals at each wavelength is measured. Away from absorption edges, the far-field diffracted signal from different harmonics will be similar, with the diffraction angle scaled by wavelength. The impact on fringe visibility of the incoherent sum of diffraction signal for a source with finite bandwidth is shown in fig 1. Fig 1a) shows the spectra of the sources, one broad-band, and one harmonic with similar envelope. Fig 1(b) and (c) show the fringe patterns from simulated Young's slits experiment illuminated with the two spectra in (a), with a slit width and separation of 1 micron. Fringes from the harmonic source, (c) retain some visibility at high scattering angles that is not apparent in the envelope case shown in (b).

This is an Open Access article distributed under the terms of the Creative Commons Attribution License 2.0, which permits unrestricted use, distribution, and reproduction in any medium, provided the original work is properly cited. 
The source would produce equivalent fringe visibility to a fully temporal coherent signal and yield a CDI solution if the incoherence is not sampled in the diffraction pattern i.e. the coherence length is longer than the object plus zero padding [3]. Experimentally it has been shown [4] that for a broad continuous spectrum, phase retrieval algorithms fail to reconstruct if full coherence is assumed.

a)

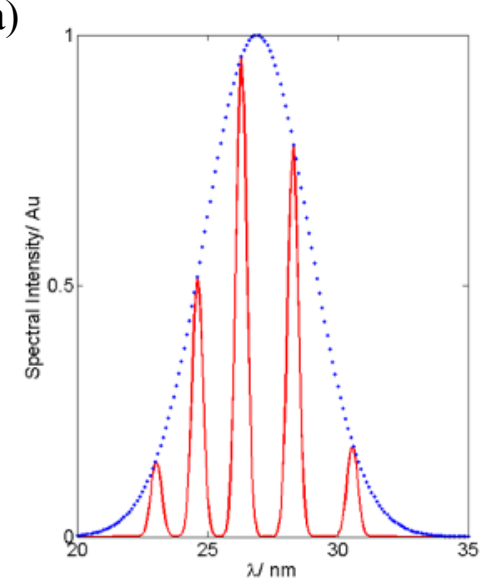

b)
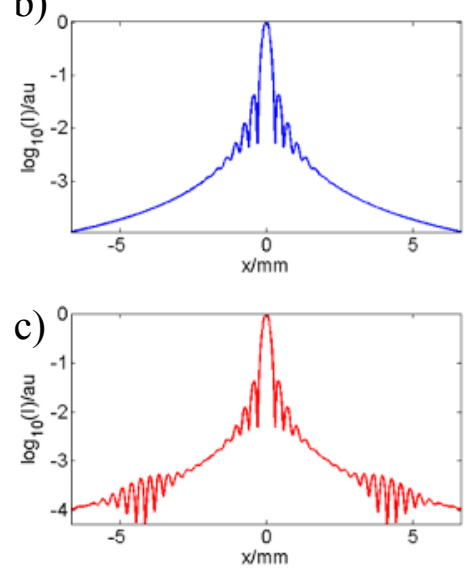

Fig 1: a) A typical high harmonic spectrum (solid line) and envelope (dotted line). The diffraction pattern from a 1 micron spacing and slit width Young's slits experiment for the envelope b) and c) the harmonic spectrum

Algorithms have been developed for both the Fresnel [5] and Fraunhofer [6] regimes using progressive updates of the CDI constraints to converge to a solution for a well-known spectrum. The differing phase matching conditions mean the high harmonic spectrum varies over the width of beam. Unless considerable prior information is known about the object's structure [6], breaking the diffraction ambiguity so that the spectrum can be recovered, the algorithm struggles to converge.

Harmonic sources possess a modulated spectrum bracketed by an envelope (figure 1(a)), which modulates the fringe visibility of the recorded incoherent sum. This signal is not a correct reciprocal space representation of the exit wave field. We show that high resolution images acquired with high temporal coherence (1.8\% rel. bandwidth) are approximated by images acquired with harmonic spectra whose envelope has $13 \%$ relative bandwidth when the latter are reconstructed assuming full temporal coherence. The reconstructions are not stable, but show more detail than would be expected for a continuous broad spectrum.

\section{Experimental Method}

Pulses of $40 \mathrm{fs}$ duration and $3 \mathrm{~mJ}$ energy centred at $800 \mathrm{~nm}$ were focussed into $40 \mathrm{mbar}$ of Argon confined to a cell, to generate a comb of frequencies down to the soft X-ray regime. Two sets of $200 \mathrm{~nm}$ thick aluminium filters were used to filter the $800 \mathrm{~nm}$ radiation from the EUV harmonics. Two experimental configurations were used: broadband, with $13 \%$ relative bandwidth, using a broadband $\mathrm{Mo} / \mathrm{Si}$ focussing optic (figure2(a)), and narrow-band, using a flat, narrowband $\mathrm{Mo} / \mathrm{Si}$ mirror in combination with the focussing optic to filter out the $31 \mathrm{st}$ harmonic $(27 \mathrm{~nm})$, with $1.8 \%$ relative bandwidth. The sample used for this experiment was an amplitude mask milled by focussed ion beam through $100 \mathrm{~nm}$ gold on $50 \mathrm{~nm}$ silicon nitride prepared by Southampton University Nanofabrication facility (figure 2(b)). The binary nature of the transmission fulfils the wavelength independent criteria. Both experiments had diffraction limited resolution of $90 \mathrm{~nm}$. 


\section{Image Reconstruction}

The reconstruction presented in figure 2(e) from the broad bandwidth data (figure 2(c) was obtained using a monochromatic HIO algorithm, unmodified except for the standard shrinkwrap [7] addition applied every 20 iterations. This reconstruction was selected as the iteration corresponding to the minimum Fourier error [7], and was repeatable for $>90 \%$ of initial random phase seeds. The monochromatic data (figure 2(d) and (f)) reached convergence after 1000 iterations of the same algorithm. Comparing the data-sets in figure 2(c) and (d), the effect of the low coherence is to lower the visibility of the spatial frequency data periodically across the detector, a different effect than that which is shown by a continuous source such as that provided by a synchrotron [4] or LP. The ambiguity due to these bands is the main reason for the lack of stable solution; enough information is contained in the high visibility data to reconstruct a poor quality reconstruction with apparently sharp features. This is only apparent in the partial coherence case presented by a high harmonic source.

a)

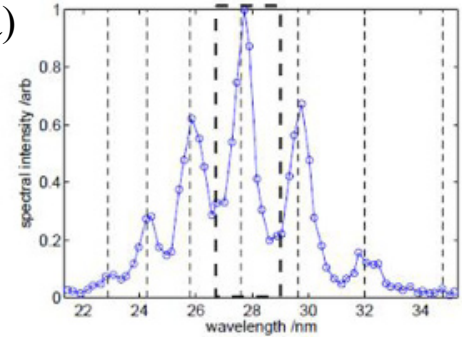

b)

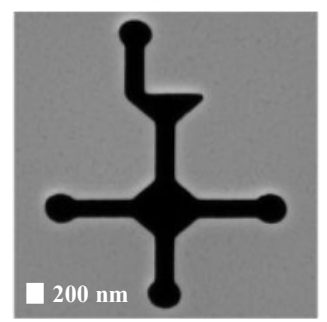

c)

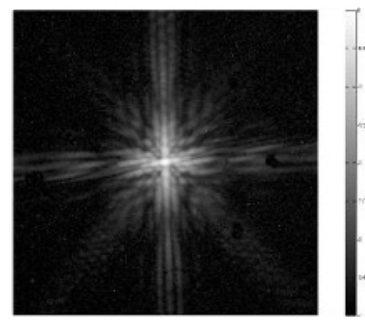

d)

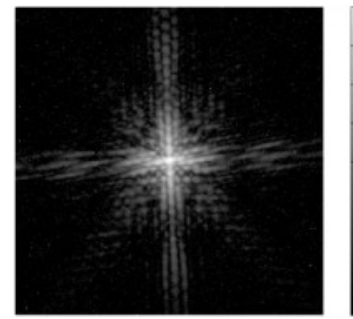

e)
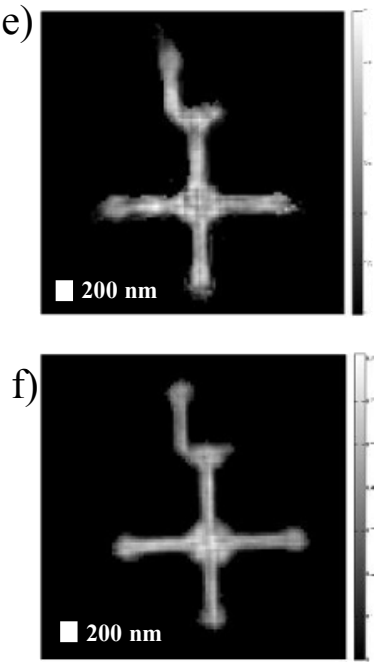

Fig 2:(a)Measured spectrum in the sample plane for the broadband experiment. The dotted line shows the harmonic selected for the narrowband experiment. (b) scanning electron micrograph of the sample.(c) scattering data and (e) reconstruction for the broadband source (d) scattering data and (f) for narrow band source.

\section{Conclusions}

We have shown that phase reconstruction using a source with a broadband harmonic spectrum is possible even where previous experiments have shown it to fail using sources with similar broadband spectral envelopes. The reconstruction is not stable, but reproduces much of the detail seen in narrow bandwidth reconstructions using a single harmonic. This result will lead to improved reconstruction algorithms and better understanding of how coherence is treated through CDI.

[1] E. Abbe, Archiv für Mikroskopische Anatomie, vol. 9, no. 1, pp. 413-418, Dec. 1873.

[2] J. R. Fienup, Optics Letters, vol. 3, no. 1, pp. 27-29, 1978.

[3] E. H. Anderson and K. O. Hodgson, Physical Review B, pp. 1-6, 2003.

[4] B. Abbey et al., Nature, vol. 5, no. July, 2011.

[5] B. Abbey et al., Nature Physics, vol. 4, no. 5, pp. 394-398, Mar. 2008.

[6] B. Chen et al., Physical Review A, vol. 79, p. 023809, 2009.

[7] S. Marchesini et al., Physical Review B, vol. 68, no. 14, pp. 1-4, Oct. 2003. 